\title{
Prolégomènes à une édition critique des poésies d'André Chénier. Écriture sensorielle et lecture auditive
}

Edouard Guitton

\section{(2) OpenEdition}

Journals

Édition électronique

URL : http://journals.openedition.org/studifrancesi/8153

DOI : 10.4000/studifrancesi.8153

ISSN : 2421-5856

Éditeur

Rosenberg \& Sellier

Édition imprimée

Date de publication : 1 mai 2009

Pagination : 77-90

ISSN : 0039-2944

\section{Référence électronique}

Edouard Guitton, «Prolégomènes à une édition critique des poésies d'André Chénier. Écriture sensorielle et lecture auditive », Studi Francesi [En ligne], 157 (LIII | I) | 2009, mis en ligne le 30 novembre 2015, consulté le 12 janvier 2021. URL : http://journals.openedition.org/studifrancesi/8153 ; DOI : https://doi.org/10.4000/studifrancesi.8153

\section{(c)}

Studi Francesi è distribuita con Licenza Creative Commons Attribuzione - Non commerciale - Non opere derivate 4.0 Internazionale. 


\section{Prolégomènes à une édition critique des poésies d'André Chénier. Écriture sensorielle et lecture auditive}

La première mission d'une édition critique consiste à recenser et examiner tous les états connus du texte considéré, puis à choisir le texte de base, les variantes fournies par les autres versions du texte étant signalées dans l'apparat critique. La discipline de l'édition critique telle qu'elle est pratiquée de nos jours «se fonde sur la tradition prestigieuse et sur les exigences rigoureuses de la philologie». La méthode philologique «est une école de rigueur et de précision, d'acribie, de sobriété et de discrétion, d'humilité enfin» ${ }^{1}$. Dans cette perspective, l'édition des textes a toutes les caractéristiques d'une science exigeante. Pourtant, dans la pratique, elle est aussi une casuistique, car chaque auteur, chaque texte, tributaires des conditions qui les ont vus naître, ont leurs particularités spécifiques et les problèmes que soulève telle ou telle œuvre ne peuvent être résolus à coup de principes généraux et catégoriques.

En matière d'édition, les poésies d'André Chénier constituent un cas exceptionnel dans la mesure où, sauf Le Jeu de paume (1791) et l'Hymne aux Suisses de Châteauvieux (1792), elles n'ont pas été imprimées du vivant de l'auteur. La mort prématurée du poète sur l'échafaud explique qu'il n'ait laissé derrière lui qu'une œuvre inachevée et inédite, donc posthume. Les circonstances complexes de la conservation et de la transmission de ses manuscrits ont été minutieusement exposées dans la récente édition de ses œuvres poétiques ${ }^{2}$. Il en ressort que les conditions nécessaires à l'établissement d'une édition satisfaisante sur le plan philologique n'ont été remplies qu'après le dépôt à la Bibliothèque nationale des papiers de Chénier et leur mise à la disposition du public en 1899. Cette étape capitale annulait en grande partie ce qui avait été réalisé auparavant, dont voici l'essentiel: en 1819, publication de l'édition Latouche des Euvres complètes, en fait une anthologie très incomplète et très insuffisamment informée et qui le resta malgré plusieurs augmentations; en 1862 (puis en 1872), publication par les soins de Becq de Fouquières de la première «édition critique», à cette réserve près que l'éditeur n'avait pas eu accès aux manuscrits détenus jalousement par Gabriel de Chénier, neveu du poète, ce qui lui a fait commettre des fourvoiements involontaires; en 1874, publication de l'édition Gabriel de Chénier qui divulgue enfin tous les documents qu'il détenait mais commet maintes bévues par défaut de compétence. Ajoutons au passif du bilan éditorial la perte des manuscrits de Chénier conservés par Latouche à la suite du pillage de sa villa en 1871: accident irréparable, qui amputait un ensemble déjà fragmentaire de sa partie la plus riche et la plus achevée (entre autres les manuscrits de l'Invention, de l'Aveugle et d'un grand

(1) Cluster 13 - Culture, patrimoine, création - Éditions critiques, pp. 1-2.

(2) ANDRÉ CHÉNIER, CEuvres poétiques, tome I, édition critique par Georges BuIsson et Édouard
GuitTon, Éditions Paradigme, 2005 (ci-après $O P 1)$. Voir les pages 5 à 13. Voir aussi la Chronologie (pp. 31-99) et les «Remarques sur cette édition» (pp. 101-106). 
nombre d'élégies). Bien placé pour en juger, Becq de Fouquières pouvait déclarer à la fin de sa vie: «L'avenir nous doit une dernière édition critique, qui, pour être définitive, exigera beaucoup de sagacité et un savoir étendu» ${ }^{3}$.

Du moment qu'on disposait du manuscrit autographe, l'établissement critique du texte semblait une opération simple à réaliser; en réalité elle était redoutablement compliquée. Au déchiffrement s'ajoutait la nécessaire mise en ordre des fragments, tâche vertigineuse. En 1905 (rééd. en 1907) paraît une édition des Bucoliques, publiées d'après le manuscrit original dans un ordre nouveau par José-Maria de Heredia qui se révèle en l'occurrence à la fois poète sensible et philologue averti comme en témoigne sa préface très remarquable intitulée «Le manuscrit des Bucoliques». De 1908 à 1919 Paul Dimoff fait paraître les trois volumes de son édition des CEuvres complètes de André Chénier publiées d'après les manuscrits: le quatrième volume consacré aux proses n'a jamais paru. Elle mériterait le qualificatif de critique quoiqu'elle limite son objet à l'établissement du texte, rejetant les commentaires historiques et littéraires dans la thèse volumineuse qui allait la suivre. Disjonction incommode qu'aggravait la méthode de classement adoptée par Dimoff: le texte, littéralement pulvérisé, devenait à peu près illisible. Le scrupuleux éditeur a laissé passer une vingtaine de fautes de lecture. En outre, respectant les principes en usage en son temps, il a pris le parti de normaliser la ponctuation et l'orthographe de Chénier, quitte à indiquer en détail dans l'apparat critique la moindre de ses interventions.

Faute de mieux, l'édition Dimoff a fait autorité jusqu'en 2005. Sur bien des points elle était devenue totalement obsolète. En un siècle le concept d'édition savante d'un texte ancien a notablement évolué. À l'omnipotence de l'éditeur autorisé à modifier ou corriger à son gré la copie de l'auteur sous couleur de la "parfaire" s'est progressivement substituée une attitude de rigueur et de modestie que résume bien le mot par lequel Jacques Robichez termine sa préface de l'édition des poésies de Verlaine: «Je n'ai pas voulu collaborer avec Verlaine». Autrement dit, interdiction de travestir la partition pour quelque motif que ce soit; souci d'offrir à l'utilisateur un instrument de travail aussi fidèle que possible et de le mettre en contact direct avec le créateur: «fiabilité du texte établi» et «authenticité de la leçon d'histoire» qui en découle sont les deux impératifs prioritaires d'une édition critique digne de ce nom ${ }^{4}$. S'agissant de ses manuscrits conservés, André Chénier ne nous a pas laissé de recueil terminé, mais ses ébauches, quoique lacunaires, se présentent sous forme de fragments soigneusement rédigés et, sauf de rares exceptions, parfaitement lisibles. «Si vous lui voulez une correction irréprochable, disait déjà Latouche, allez le redemander au tombeau qui se ferma sur lui à trente et un an» ${ }^{5}$. On serait tenté d'ajouter: prenez-le tel quel, scrutez ses particularités, interrogez-vous sur ses intentions au lieu de le modifier au risque de le défigurer. Quand d'une œuvre à éditer ne subsiste qu'un manuscrit autographe, les seules variantes recevables sont internes au manuscrit: toute suscription due à une main étrangère et non conforme à la volonté de l'auteur est à rejeter. Qu'il s'agisse de l'information historique ou de la philologie proprement dite et de ses annexes (stylistique, phonétique, linguistique, papyrologie, codicologie, etc.), qu'il s'agisse de la matérialité du texte ou de son interprétation, le progrès des connaissances et l'évolution des mentalités ont fait naître une exigence d'authenticité qui incitait à ramener l'ouvrage à son état originel au lieu de le tirer vers un avenir auquel il était étranger. Il s'en est suivi dans toutes les disciplines de l'esprit un décapage sans précédent pouvant mener à la redécouverte d'un

(3) Cité dans OP1, p. 12

(4) Cluster 13, loc. cit., p. 2.
(5) Euvres complètes d'André Chénier, Paris, 1819 , in- $8^{\circ}$ (ci-après L1819), p. XVIII. 
état primitif oublié. Le phénomène est particulièrement flagrant en musique et en architecture, mais il concerne également la littérature des XVII et XVIII ${ }^{\mathrm{e}}$ siècles comme le prouvent les éditions les plus recommandables publiées depuis une cinquantaine d'années en ce domaine.

Ce souci de fidélité «textuelle» doit-il exclure ou inclure la ponctuation? La ponctuation fait-elle oui ou non partie intégrante du texte que l'on édite? Hic grammatici certant. Répondre par la négative signifierait qu'elle n'est qu'un ornement accessoire que chaque utilisateur peut modifier à son gré. Cette attitude n'est plus de saison. Osons le dire: elle n'est pas scientifique. Longtemps les typographes se sont cru autorisés à «corriger» la copie originelle avant de l'éditer, si bien que le lecteur n'était jamais sûr d'avoir sous les yeux le texte tel que l'auteur l'avait conçu. Les progrès du savoir érudit ont permis de placer dans une perspective évolutive et historique tout ce qui ressortit à la graphie d'un texte. Née sous sa forme moderne au XVI ${ }^{\mathrm{e}}$ siècle, la ponctuation des écrivains, à la différence de celle des typographes, n'avait jamais obéi à un système rigide et intangible avant qu'elle ne devienne, dans le courant du XIX ${ }^{e}$ siècle seulement, un objet d'enseignement draconien appris sur les bancs des écoles. L'amélioration de l'écoute des textes a fait découvrir que, loin d'être seulement un instrument de police syntaxique, les points, les virgules et autres signes diacritiques concouraient, surtout en poésie, à la scansion des phrases dont ils ne pouvaient être disjoints. Cette découverte ne date pas d'aujourd'hui. Dès avant 1950, les éditeurs de Chateaubriand, en se fondant sur l'édition originale des Natchez ou sur les manuscrits des Mémoires d'outre-tombe, établissaient que la ponctuation de l'auteur, «plutôt qu'aux règles de la logique et de la grammaire, obéit aux suggestions de l'harmonie; elle est moins faite pour l'œil que pour l'oreille». Jean Mourot fondait sur leurs enquêtes son étude très fouillée du Génie d'un style. Il déclarait: «J'ai pu me convaincre que les particularités de la ponctuation de Chateaubriand éclairaient tous les aspects de son rythme». A la même époque et pour des raisons analogues, Jean Fabre éditant Le Neveu de Rameau disait qu'il lui avait paru nécessaire

de conserver, dans ses extravagances même, la ponctuation de Diderot. Celle-ci n'obéit pas, en effet, aux règles de la grammaire ou de la logique, mais à celles de l'expression dramatique et quasi musicale. Elle marque les accélérations, note les soupirs et les pauses, décompose et scande les pantomimes, suggère les intonations; elle est comme la respiration des personnages, la palpitation de la vie. On ne pourrait la modifier sans trahir l'expressionnisme essentiel à l'art de Diderot ${ }^{7}$.

Ce verdict on ne peut plus net a fait école; l'un des plus récents éditeurs du Neveu (en édition de poche!) déclare en écho à Jean Fabre: «L'orthographe du manuscrit a été modernisée, ainsi que les signes marquant les dialogues. La ponctuation en revanche a été scrupuleusement respectée: elle marque le rythme du texte, sa respiration même» ${ }^{8}$.

Ni Diderot ni Chateaubriand ne sont en la matière des cas isolés: la pratique d'une ponctuation rythmique et prosodique est un fait d'époque qui court du XVII au début du XIX ${ }^{\mathrm{e}}$ siècle. Racine et son théâtre eux-mêmes en fournissent la démons-

(6) Jean Mourot, Le Génie d'un style. Chateaubriand, Rythme et sonorité dans les Mémoires d'outre-tombe, Paris, A. Colin, 1960. Voir en particulier les pp. 18 à 21 d'où sont extraites nos citations.

(7) Denis Diderot, Le Neveu de Rameau, édi- tion critique avec notes et lexique par Jean FABRE, Genève, Droz, 1950, rééd. 1963, Introduction, pp. xxvi-xxvii. Ici et plus loin, les mots en italique sont soulignés par nous.

(8) Pierre Chartier, édition du Neveu de Rameau, Paris, Le livre de poche, 2001, p. 16. 
tration: en 1999 la prestigieuse collection de la Pléiade a publié une édition de ses tragédies due à Georges Forestier qui, en l'absence de manuscrits, fonde l'établissement du texte sur les tout premiers tirages, lesquels, destinés aux comédiens et pas encore au public, s'accordaient à une lecture à haute voix, récitation ou déclamation. Il en ressort, exemples à l'appui, que la ponctuation y exerce une fonction rythmique, qu'elle sert, par la présence ou l'absence de certains signes, par l'usage particulier qui en est fait, à marquer les tons et les intensités. Ce «système de notation vocale», selon lequel le poète ponctue «en dehors de la syntaxe et du sens», «relevait de règles stables, quoique différentes des nôtres». Le résultat dérange un tant soit peu nos habitudes de lecture, mais il nous invite «à lire un texte de théâtre de cette époque selon la manière même dont il a été conçu» et selon la fin à laquelle il était destiné: non pas une lecture silencieuse, mais une récitation à haute voix ou encore une déclamation?

Tout ce qui vient d'être dit de Racine, de Diderot et de Chateaubriand praticiens d'une écriture lyrique s'applique a fortiori à l'œuvre poétique d'André Chénier: ces écrivains, auxquels on pourrait joindre Jean-Jacques Rousseau et Bernardin de Saint-Pierre, appartiennent à la même mouvance esthétique, ils ont été formés à la même école et, pour reprendre les termes de Jean Fabre, l'expressionnisme essentiel propre à cette période concerne souverainement l'art de Chénier. Dès lors, on peut être surpris qu'à ce jour aucun éditeur n'ait été tenté de jouer le jeu de l'authenticité à partir du trésor inestimable et rarissime que constituait le manuscrit autographe du poète, gage unique de véracité antérieur à l'intrusion déformante des typographes et autres "correcteurs". Sainte-Beuve, en 1839, ressortit ébloui de «l'atelier du fondeur» et rêva un moment d'un projet d'édition idéale sans donner suite. Éditeur des Bucoliques après 1900, Heredia connaît fort bien les règles d'emploi de la ponctuation à l'époque classique en poésie:

Le poète se sert de ces signes pour indiquer la façon dont il entend que ses vers soient scandés, le mouvement plus rapide ou plus lent, les pauses, le prolongement, les arrêts de la diction.

Il dénonce, exemples à l'appui, les éditeurs qui se sont «fait un jeu d'altérer la ponctuation d'André Chénier» dont il dit qu'elle est «singulièrement personnelle; à la fois méticuleuse et sommaire» et, dans tous les cas, comme il le montre fort bien, justifiée par des intentions d'ordre stylistique ou prosodique. Aussi se flatte-t-il d'avoir «toujours respecté, à moins qu'elle ne fût manifestement insuffisante ou fautive, la ponctuation du poète» ${ }^{10}$. Hélas! dans la réalité, la restriction l'emporte trop souvent sur le respect affiché et l'infidélité a presque toujours le dessus. Heredia, il est vrai, était mort avant la publication de l'ouvrage: il a pu être trahi par ses éditeurs.

Paul Dimoff pousse l'inconséquence plus loin encore. Il reprend et prolonge les remarques d'Heredia: la ponctuation d'André Chénier, note-t-il,

sert en général beaucoup moins à préciser le sens des vers qu'à en rythmer l'allure. Les virgules sont presque toujours omises, quand elles n'ont pour rôle que de séparer des substantifs et des verbes réunis dans une énumération que le poète destine à être lue d'un seul trait. Au

(9) Voir, dans l'édition des Euvres complètes de Racine, I, Théâtre-Poésie, Paris, Éditions Gallimard, 1999, les pages LIX-LVIII intitulées «Lire Racine». Plusieurs travaux plus récents ont prolongé la discussion, notamment l'ouvrage d'Alain RIFFAUD, La ponctuation du théâtre imprimé au XVII siècle, Genève, Droz, 2007, pp. 228. Etude très dé- taillée qui fait ressortir l'unicité, en son temps, du cas Chénier dont le passé ne nous a transmis aucune version imprimée du vivant de l'auteur (sauf deux exceptions mineures: voir plus haut, p. 1).

(10) ANDRÉ ChÉNIER, Les Bucoliques publiée d'après le manuscrit original par José-Maria DE HEREDIA, Paris, 1907, pp. XXII-XXIV. 
contraire, des points et virgules, des points, apparaissent sans cesse dans une même période, là où l'usage courant réclamerait une simple virgule: ils arrêtent et interrompent le vers, le brisent parfois en trois ou quatre tronçons et soulignent ainsi l'émotion d'un personnage qui parle, ou marquent les divers moments d'une action. Bref, cette ponctuation nous fait mieux voir ce qu'a eu de personnel la versification de Chénier et comment ce puissant artiste a devancé en bien des endroits les romantiques dans leurs audaces ${ }^{11}$.

On a peine à comprendre qu'après une aussi remarquable analyse, qui avait le mérite de relier les détails de la graphie aux effets stylistiques, psychologiques, dramatiques du texte tels que les avait voulus l'écrivain, Dimoff n'ait pas cru de son devoir de maintenir des particularités aussi expressives; il en étendait le principe aux fragments en prose dont la ponctuation, disait-il, «contribue fréquemment à rendre sensible une intention de l'auteur, à montrer la valeur d'une expression» (ibid., p. XXIV). Malheureusement et malgré des assurances de respect scrupuleux, l'éditeur a cédé en permanence à une manie rectificatrice qui revenait à annuler l'originalité si justement proclamée du poète, quitte à signaler en bas de page les innombrables leçons corrigées. Autrement dit, la dictature de «l'usage courant», qu'il fût d'origine académique ou scolaire, l'a emporté sur toute autre considération: comme si cet usage courant était infaillible et intangible! Nous savons mieux aujourd'hui qu'il y a cent ans à quel point l'usage, comme le goût, est une notion évolutive et relative à ses conditions d'application. L'honnêteté éditoriale consiste donc à respecter l'usage courant d'un auteur et d'une époque et à en élucider les fondements au lieu de leur en imposer un qui leur est étranger.

La mise au point de Dimoff avait au moins le mérite de ruiner l'hypothèse de négligences d'écriture dues à la hâte ou à la distraction du rédacteur: cette hypothèse, insoutenable pour quiconque regarde les choses de près, se heurte à de constants démentis ${ }^{12}$. Elle ne rend pas compte de la fréquence et du nombre de ces prétendus oublis, ni de leur caractère répétitif sur toute la période créatrice du poète, des textes du collège à ceux de la prison. Un inventaire détaillé de toutes ces occurrences dépasserait les limites d'un simple article et surtout il garderait un aspect mécanique sans rapport avec le dynamisme qui préside au travail du rimeur. Nous analyserons plus tard un certain nombre d'exemples probants. Signalons pour l'instant le seul cas des juxtapositions dépourvues de signes de liaison (épithètes, substantifs, verbes) du type "mourante échevelée" (IPP III-b, OP1, p. 113). On en rencontre dès les essais de jeunesse; on en trouve dans les Élégies («l'insomnie amère impatiente-pourquoi pourquoi les dieux - o Camille Camille»); on en trouve en abondance dans les Bucoliques («nos amours tremblantes incertaines-A la jeune Mnaïs rendez rendez hélas-Et l'amour l'amour seul-Pleure pleure c'est moi pleure», etc.); on en trouve encore à une date tardive et jusque dans la prison («Le feu le fer arment mes mains -- Vienne vienne la mort»). Chénier écrit aussi: «Fuis ne me livre point — Elle a vécu Myrto — Lui grands Dieux!» Négligences? distractions? incorrections? ou au contraire recherches expressives volontaires, effets rythmiques intentionnels? Un examen attentif des faits confirme le diagnostic de Heredia et de Dimoff: ici et ailleurs la main de l'auteur laisse sentir sa présence en chacune des nombreuses «licences» qu'il s'autorise et, si la main rédige, c'est l'oreille qui la conduit. Avoir de l'oreille et avoir du cœur: telles

(11) Euvres complètes de André Chénier, 3 voll., Paris, Delagrave, rééd. 1966 (ci-après D.), I, pp. XXIII-XXIV.

(12) Le «J'oublie toujours quelque chose» cité dans OP1, p. 103, ne concerne pas la ponctuation, mais le rassemblement des idées, donc l'invention proprement dite, ce qui est beaucoup plus logique et vraisemblable. 
sont les deux conditions sans lesquelles nul ne deviendra poète, tous le répètent à qui mieux mieux à l'époque de Voltaire. Il faut à ce propos rectifier une idée reçue tout à fait fausse selon laquelle la poésie du XVIII ${ }^{\mathrm{e}}$ siècle est dénuée de sensibilité et se contente d'appliquer servilement des procédés mécaniques de composition. L'esthétique classique se fait une haute idée de la poésie, ce «langage des dieux» qui se distingue radicalement de la prose. Chacune, «pour dire la même chose, a sa manière qui ne ressemble point à l'autre» ${ }^{13}$. La prose donne la priorité au sens et au message rationnel qu'elle doit transmettre au destinataire. La poésie, en revanche, existe d'abord par les sons et c'est en quoi elle s'apparente à la musique: à la limite, il n'est pas absolument nécessaire de comprendre la signification d'un vers pour en apprécier la beauté. C'est par l'barmonie du style, notion capitale sur laquelle s'étendent longuement les poéticiens, que la poésie exerce sur l'auditeur un charme dont elle a le privilège exclusif. Ce sont les sons qui modèlent le sens d'un vers et non l'inverse: Paul Valéry ne cessera de le suggérer. On ne dira pas pour autant que le son élimine ou éclipse le sens: le rationalisme classique ne peut accepter l'idée d'une poésie dénuée de sens et André Chénier, dans l'Essai, s'en prend avec vivacité à l'homme sans génie qui, lisant les grands poètes, ne capte que l'enveloppe sonore sans atteindre le fond:

C'est un vain bruit, c'est le nombre, la cadence, le rythme qui plaît à son oreille. Des mots pris au hasard et arrangés harmonieusement sur les mêmes mesures, sans produire aucun sens, lui feraient le même plaisir.

Il imite mécaniquement les procédés propres à la poésie «et il croit avoir peint quelque chose, et il se dit qu'il est bien beau d'être poète» alors qu'il n'est capable d'enfanter qu'un fœtus informe ${ }^{14}$. Au contraire, seul le génie doué d'une «imagination pénétrante et vive» sait que l'alchimie poétique ne sépare pas le sens et le son: tous deux tels des jumeaux indissociables contribuent à créer la vraie poésie et, comme il est dit dans L'Invention,

Tout s'allie et se forme et tout va naître ensemble.

(D. II, p. 25)

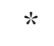

$* *$

Le caractère prémédité de la manière dont le poète a établi son texte étant attesté, reste à expliciter les mobiles de ce comportement et à en éclairer les effets. Les mobiles sont à la fois extérieurs ou externes, relatifs à l'environnement culturel au sein duquel se forgent les mentalités, et internes, propres à l'individu concerné. Le jeune André, comme tous ses contemporains, ceux du moins qui allaient à l'école, a reçu au collège une éducation très soignée qui incorporait l'apprentissage de la versification, lequel se poursuivait ensuite dans des groupes de travail (nous dirions des «séminaires») que rassemblaient autour d'eux les maîtres de l'heure, par exemple Louis Racine, Marmontel ou Le Brun. Quel entraînement y subissait-on? Priorité à l'écoute et à l'oreille: tel est l'impératif catégorique, inlassablement répété par les théoriciens et les praticiens de la poésie, à tout le moins de Jean-Baptiste Rousseau ou de Rollin, l'auteur du Traité des Études, à Chateaubriand: «Il m'apprit à respecter l'oreille», dit ce dernier de Fontanes, qui était un brillant représentant de l'école

(13) Clément, vers 1760. Cité par JeAn FabRe, Chénier (ci-après CH), Paris, Hatier, 1965, p. 159.

(14) Euvres inédites de André Chénier publiées par Abel LeFranc (ci-après $A L$ ), Paris, 1914, pp. $115-117$. 
française de poésie sous Louis XVI. Le siècle dit des Lumières a mis dans son programme un examen systématique des sons de la langue française afin de la guérir de ce qui est ressenti comme une infirmité par comparaison avec les idiomes anciens (le grec et le latin), voire modernes (surtout l'italien): sa platitude, l'absence de relief et de couleur qui la caractérise. Dans cette entreprise se distinguent pour la réflexion théorique l'abbé d'Olivet avec son Traité de la prosodie française (1736) et Marmontel avec sa Poétique française (1763) récupérée dans ses Éléments de Littérature (1787); pour la mise en pratique les traducteurs, en tête desquels se trouve Jacques Delille, tiennent le devant de la scène. Savoir faire résonner les longues et les brèves à l'instar des poètes grecs et latins afin de «donner à l'alexandrin français les ressources de l'hexamètre» (CH, 1965, p. 174) suppose une longue fréquentation des modèles et un apprentissage adéquat. Á quoi tient que les clausules de la poésie grecque ou latine charment l'oreille avec une force que les traducteurs ne parviennent pas à reproduire? Cette question obsède les esprits ${ }^{15}$. Or, c'est à partir des poètes latins et grecs que le débutant apprend à versifier en français: ainsi a fait le jeune André dès le collège, comme tout le monde. Il avoue dans une de ses notes philologiques, à propos de deux vers extraits d'une idylle de Théocrite où le mot grec $\dot{\alpha} \delta v$ est quatre fois répété: «Depuis mon enfance, la douceur et la simplicité de ces deux vers ont eu pour moi un charme qui m'attendrit et que je ne puis expliquer» ( $A L$, p. 221). On touche là à l'origine d'une vocation. Plus généralement, c'est la sensibilité aux bruits de la nature qui caractérise le poète, ou le musicien, à l'état naissant. Très parlante à cet égard est l'élégie I, 17 (OP 1, p. 220-221); saluant le retour de ses Muses, le poète les invoque en ces termes:

Ô sons! ô douces voix chères à mon oreille,

Ô mes Muses, c'est vous.

Muses et sons se confondent et du son à la voix il n'y a qu'un pas. Autrement dit, la sensation est l'élément porteur de l'inspiration: conséquence logique de l'essor du sensualisme après 1750 . L'oreille, la voix occupent une place prépondérante dans la poétique d'André Chénier et l'on comprend pourquoi. On comprend aussi qu'une poésie aphone soit ressentie comme inexistante: la poésie «langage sonore» est faite pour être récitée et entendue. Un Delille, un Roucher magnétisaient l'auditoire lors de leurs lectures publiques. Une fois le texte imprimé et lu en silence, le charme retombait. De quel côté la vérité de la poésie se situait-elle? dans la lecture à voix basse ou dans la récitation à voix haute? Joubert n'avait pas tort lorsqu'il disait: «L'abbé Delille n'a dans la tête que des sons et des couleurs; mais voyez l'usage qu'il en fait» ${ }^{16}$. Cette réduction aux sensations était dans la droite ligne d'un sensualisme devenu dominant après 1760 et dont Chénier a forcément subi l'impact tout en refusant les excès auxquels il donnait lieu.

«L'originalité d'André Chénier ne peut être appréciée que par rapport à la pratique des poètes de son temps»; il faut donc libérer sa poésie «de normes et de critères qui n'étaient pas faits pour elle» (J. Fabre, CH, 1965, p. 281). Salutaire mise en garde contre les anachronismes! Quand il débute, vers 1780, le jeune homme trouve à sa disposition un art poétique à la fois bien structuré et en pleine évolution. La fidélité aux rimes et aux mètres ne souffre pas de discussion; rejets, enjambements, figures

(15) Voir à ce sujet É. GuitTon, Ponctuer ou scander; réciter ou déclamer, «Revue de Littératures $09 »$, Vallongues, pp. 115-118.
(16) Pensées de J. Joubert, éd. P. De Raynal, Paris, Didier, 1880, II, p. 383. 
de style, harmonie imitative font partie de l'outillage usuel du rimeur et concourent à l'animation du discours: la «métromanie» est à son comble. Mais tout ce qui ressortit à un mécanisme mimétique doit être dépassé. Une nouvelle poésie s'édifie dont la nouveauté va venir en particulier de l'apport d'une troisième dimension des mots tenant à leur sonorisation. Le renouvellement du vers français dans la seconde moitié du XVIII ${ }^{\mathrm{e}}$ siècle provient moins d'une dislocation, comme on l'a cru longtemps, que d'un phénomène d'assouplissement et d'étirement, et de sa répercussion sur la résonance des vers: plus la corde de l'instrument est tendue, plus elle vibre. Tout ce qui concerne les rapports qu'on peut trouver entre l'harmonie du style et l'harmonie musicale intéresse l'élite pensante de l'heure, en particulier Marmontel qui est à la fois homme de lettres et musicologue. Une évolution parallèle affecte la poésie et la musique. Les premiers essais de Chénier coïncident avec la querelle des Gluckistes et des Piccinnistes: le jeune homme, qui est féru d'opéra, ne cache pas sa préférence pour la musique à l'italienne, celle qui évolue vers un art vocal libéré, le fameux bel canto (voir $O P 1$, p. 195 et 275). Une libération analogue affecte l'art poétique à l'intérieur de ce qui constitue deux de ses principes fondamentaux, le mouvement (et son corollaire le tempo) et l'expression (et son corollaire les nuances). A la recherche d'un vers non pas libre mais libéré, ainsi apparaissent les poètes français après 1770: Roucher, Fontanes parmi d'autres pourraient être cités en exemple. La ponctuation elle-même, qui est une des modalités de ces efforts libérateurs, n'échappe pas à cette émancipation, marque d'un expressionnisme résolument neuf: une ponctuation elle aussi libérée se rencontre dans les publications du moment ${ }^{17}$. Le point culminant de cette tendance se situe entre 1780 et le début de la révolution de 1789.

André Chénier, qui est par tempérament un «oseur» et dont la sensibilité aux sons est éclatante, devait se porter spontanément en tête du peloton des inventeurs dans le domaine spécifiquement linguistique de l'écoute syllabique des vers, sous la double férule de la métrique et de la prosodie. Sur ce point comme sur tant d'autres, l'avancée apparente coïncide avec un retour aux sources. Le poète aspirait au fond de lui-même à retrouver la nudité des Anciens et à pratiquer une écriture débarrassée de toute scorie. D'où l'absence possible de toute ponctuation. Nous en donnerons des exemples dans un article à venir.

Il peut arriver à l'inverse, comme Heredia et Dimoff l'avaient très bien remarqué, que la ponctuation ait un rôle de renforcement ou de soulignement. Tel vers isolé des Bucoliques ponctué de la manière suivante:

Des vers enfants des bois. Nés sons, fils de l'ombrage.

(D. I, p. 246)

surprend au premier abord. Le premier hémistiche termine une phrase; le second en ouvre une nouvelle, qui s'interrompt aussitôt. C'est la définition lapidaire d'un idéal sonore à coloration pastorale et d'une fusion entre les éléments de la nature et une inspiration spécifiquement sensorielle; l'alexandrin est ici de type gnomique, il affirme ou constate. De même sont soigneusement ponctuées les invocations, salutations ou déclinaisons d'identité, par exemple le célèbre Salut aux Dieux de l'Euxin (OP1, p. 285) ou, dans les Bucoliques, l'invocation à Proserpine (D. I, p. 22). Ou bien telle réplique adressée par un personnage à d'autres:

Pleurez, ô mes amis; adieu, je ne suis plus.

(D.I, p. 276)

(17) Voir notamment le curieux poème intitulé Des Genres poétiques qui, paru en 1790 et anonyme, présente d'étonnantes ressemblances avec L'Inven- tion. Lire à son propos É. GuITTON, Pbysionomies d'A.C., pp. 33-40. 
Alexandrin auquel sa triple césure imprime une tonalité pathétique haletante. Ou encore le rythme lancinant de marche funèbre, comparable au style louré des musiciens, que l'on rencontre au début de La Jeune Tarentine. Si, en revanche, André Chénier écrit:

Viendra, nouvel Homère,

Faire encor disputer sur sa noble origine

Colophon Smyrne Rhode Athènes Salamine.

l'absence de virgules au dernier vers se justifie par la coloration du propos: les noms des cités énumérées forment comme une frise sculptée au fronton d'un temple; l'effet rythmique et mélodique est assez net pour qu'on doive le respecter.

Ainsi l'acoustique, science à la variabilité infinie, se mettait-elle au service de la poésie. Dans ce travail de subtile précision, chaque détail est parlant, la moindre impulsion mérite d'être interprétée. Cette variabilité illustre la plasticité du matériau sonore utilisé par le versificateur. Les cas extrêmes requièrent particulièrement l'attention. Quand il supprime toute ponctuation, André Chénier rejoint partiellement Apollinaire déclarant un siècle et demi plus tard:

Je ne l'ai supprimée que parce qu'elle m'a paru inutile et elle l'est en effet, le rythme même et la coupe des vers voilà la véritable ponctuation et il n'en est point besoin d'une autre ${ }^{18}$.

Les deux poètes se livrent en l'occurrence à la même expérience fondamentale qui, loin d'inventer des recettes d'avant-garde, retrouve au contraire les lois primitives d'une rythmique élémentaire, celle de la marche et du pas cadencé (tous deux composent «en marchant»), mais Chénier est moins systématique dans l'application qu'il en fait, peut-être parce que son exigence auriculaire est plus forte: elle veut nuancer le plus ou le moins d'effet donné au vers et éviter ainsi le risque de monotonie provoqué par l'emploi systématique du même procédé. La perfection qu'il recherche, c'est en marge de Malherbe qu'il l'a le mieux définie quand il déclare à propos des Larmes de saint Pierre:

Quoique le fond des choses soit détestable dans ce poème, il ne faut point le mépriser: la versification en est étonnante. On y voit combien Malherbe connaissait notre langue et était né à notre poésie; combien son oreille était délicate et pure dans le choix et l'enchaînement de syllabes sonores et harmonieuses, et de cette musique de ses vers qu'aucun de nos poètes n'a surpassée $e^{19}$.

«Le choix et l'enchaînement de syllabes sonores et harmonieuses» (Chénier) et «le rythme même et la coupe des vers» (Apollinaire) se rejoignent pour définir les cadences et les modules inhérents au langage poétique indépendamment des ornements surajoutés après coup aux vocables qui le constituent.

La sonorisation du langage propre à la poésie est, on le voit, loin d'être homogène et automatique: toute poésie ne résonne pas de la même manière. Le champ d'expansion offert à l'esprit inventeur est en pleine reconversion. À la traditionnelle distinction des genres l'âme sensible tend à substituer une distinction des styles, concept indivis entre la rhétorique et la musique auquel Antoine de Cournand consacre

(18) Cité par M. DÉCaudin dans Le Dossier d' «Alcools», Paris, Droz-Minard, 1965, p. 40.

(19) ANDRÉ CHÉNIER, Euvres complètes publiées par Gérard Walter, Paris, Bibl. de la Pléiade (ciaprès $W$.$) , p. 803$. 
en 1780 un poème que son auteur ose présenter comme le nouvel Art poétique du siè$\mathrm{cle}^{20}$. La préface s'ouvre sur une mise à distance de Boileau et se termine par un appel à ce qu'il faut bien appeler l'originalité en art. Deux préceptes s'imposent au poète selon Cournand: étudier la nature, réservoir d'images vraies, et laisser parler son génie individuel. Le découpage de l'espace en quatre secteurs, le simple, le gracieux, le sublime et le sombre, valorise l'approche sensitive du réel. Une redistribution des anciens genres illustre cet infléchissement: la fable et la pastorale appartiennent au Simple dont la faculté maîtresse est la naïveté; Pindare, l'épopée, la tragédie, ainsi que les spectacles grandioses de la nature, prennent rang dans le Sublime; l'élégie, la mort et les tombeaux occupent le Sombre. A chaque type de poésie correspond un ton, ou une tonalité spécifique. À chaque poète de choisir la tessiture qui convient le mieux à son tempérament:

Son style sera l'image des affections de son âme; douces ou fortes, elles viendront se peindre dans ses vers comme d'elles-mêmes. Ainsi l'impression de la main fait rendre à un instrument tous les tons nécessaires à un bel accord; mais le premier mobile est dans l'âme du musicien. (Les Styles, fin de la préface, pp. XXXIII-XXXIV).

Ces formules remarquables, tout en poussant à l'extrême l'assimilation de la poésie et de la musique, soulignent à la fois la pluralité des modes d'expression et l'unité constitutive de l'inspiration.

Une pareille analyse nous incite en dernier ressort à porter intérêt à la récitation qui est l'enjeu ultime de la création poétique: comment se fait le passage de l'énonciation à la prononciation, telle est la question. Nous manquons de témoignages sur la façon dont André récitait ses vers, mais ce que l'on sait de cet exercice à son époque incite à penser que le résultat était plus proche de la déclamation ou du chant que d'une lecture monocorde. L'interprète adaptait son instrument aux exigences de la partition et une mise en scène s'imposait. André Chénier parle dans l'Épître sur ses ouvrages du «bruit des vers psalmodiés», ce qui renvoie à une diction intermédiaire entre la parole et le chant. Ici encore, le rapprochement avec Apollinaire est éclairant: l'auteur d'Alcools «avait enregistré plusieurs de ses poèmes pour les Archives de la Parole en 1913: chaque vers est isolé par un silence, et dit d'un seul souffle, comme chanté sur un air de mélopée» ${ }^{21}$. Il est à penser que Chénier, lui, variait le ton suivant le type de poésie récitée. Le chantre des Bucoliques fait germer pour sa «jeune Poésie» des vers mélodieux, [...] des vers doux, sonores, liquides. (D. I, p. 4). Cette modalité à dominante aquatique convient à un registre décontracté, ludique, pastoral, où le débit vise à la transparence. Pour l'élégie qui réclame Un vers brûlant d'amour et de larmes trempé (OP1, p. 200) les Muses ont amolli nos sons (OP1, p. 243). Pour une inspiration plus passionnée le poète annonce à plusieurs reprises des vers tumultueux: échauffement, bouillonnement, embrasement provoquent les transports de l'âme caractéristiques d'un état qui peut confiner à la folie (notamment en cas de fureur érotique). Fluidité d'une part, incandescence de l'autre, dans les deux cas les règles d'une syntaxe ordinaire sont débordées au profit d'une diction appropriée. Ainsi se définissent une poétique de l'eau (vers liquides) et une poétique du feu (vers torrides) qui l'une et l'autre supposent le recours à une versification soit relâchée, soit débridée et dans les deux cas proche de l'improvisation et pour lesquelles les rigidités d'une ponctuation régulière comme un métronome se révèlent hors de saison. 
En revanche, lorsque le propos se fait didactique ou narratif, lorsque le poète argumente, défend ou attaque, enseigne ou raconte, l'agencement des mots se rapproche peu ou prou de la discipline de la prose. On ne s'étonnera donc pas de rencontrer dans les poèmes, les satires, les épîtres, les fragments dramatiques une intonation plus impersonnelle, plus discursive en général que dans les Élégies ou les Bucoliques puisque le discours y parle moins aux sens qu'à l'intellect, ainsi qu'une ponctuation moins «irrégulière». En théorie, chaque «genre» devrait avoir sa rhétorique spécifique; dans la pratique, les interférences sont fréquentes, ce qui ne simplifie pas les choses. Une attention particulière doit être portée à ce qu'il est convenu d'appeler la poésie lyrique, épithète dont la portée est en pleine mutation dans les années 1760-1780. D’après l'étymologie et par référence à son origine grecque, le poème lyrique était «non seulement chanté, mais composé aux accords de la lyre» et son mode d'expression était l'ode, mot qui en grec signifie chant. Par la suite, la situation a dégénéré: «On a dit, Je chante, et on n'a point chanté; on a parlé des accords de la lyre, et on n'avait point de lyre» $\gg^{22}$. Pourtant, les expressions de poésie lyrique ou de théâtre lyrique se sont imposées chaque fois que la connivence de la poésie et de la musique le justifiait. L'adjectif tend à désigner moins un genre particulier qu'une certaine forme de vibration chantante sensible au niveau du discours et provoquée en général par l'émotion. Après 1750 , les progrès de la sensibilité et le culte naissant de la personnalité ont acheminé le mode lyrique vers ce que les temps modernes allaient en faire jusqu'à le confondre parfois avec la poésie elle-même. Notons que le substantif lyrisme n'apparaîtra dans la langue française que vers 1830, en plein épanouissement du romantisme ${ }^{23}$.

André Chénier est au cœur de cette évolution, son œuvre incarne le passage d'une poésie dont la divinité tutélaire se nomme l'Harmonie, dont Louis Racine et Le Franc de Pompignan parmi d'autres ont célébré le culte, à une poésie que l'exemple italien pousse à placer sous le signe de la Mélodie. C'est aussi l'âge d'or de la romance; les cantiques, les psaumes, très en faveur sous le règne de Louis XV, font place à la chanson, aux récitatifs, aux airs et aux ariettes. Le hiérodrame, auquel s'apparente $S u$ zanne, fait une brève apparition. Les Bucoliques consacrent la fusion de la poésie et de la musique et se présentent comme une sorte de chant perpétuel ainsi qu'en témoigne le vocabulaire: dès le prologue sont nommés chants bucoliques, chansons, chanteurs divins, doux sons de la flûte bocagère (D. I, p. 301), tandis que l'épilogue récapitule délices.

Voilà ce que je chantais en voyageant... et ces chansons remplissaient mes voyages de

et dit adieu aux rustiques chalumeaux, aux douces chansons et à leurs sons enchanteurs. La présence des formes musicales est constante dans le recueil: chants du gondolier (Il aime les chansons, il chante), rustiques chansons des bergers et bergères, vers syracusains et grecques chansons, concerts du jeune berger dont la Muse échevelée fait alterner les doux accents et les vives chansons; rhapsodie du berger poète dont les chants savent tout peindre. Sur un registre plus élaboré, complainte de Néère où se suivent le récitatif et l'air comme il est de règle à l'opéra. Effet comparable avec «la Jeune Tarentine» où l'on reconnaît les rythmes et les accents du thrène ou de la thrénodie, chant funèbre qui remonte à la poésie grecque antique. Au point culminant du parcours, L'Aveugle met en scène le grand vieillard qui

(22) Marmontel, Éléments de littérature, article «Ode».

(23) Voir à ce sujet mon article Entre l'ancien et le nouveau: André Chénier poète lyrique, dans L'Éloge lyrique, sous la direction d'Alain GÉNETIOT, Presses Universitaires de Nancy, 2008, p. 285-303. 
en images hardies

Déployait le tissu des saintes mélodies.

Ce tissu rappelle le «tissu d'hiéroglyphes entassés les uns sur les autres» par lequel Diderot définissait la poésie dans sa Lettre sur les sourds et muets. Entassement ou alignement sans couture visible, la métaphore du tissu figure admirablement l'écoulement ininterrompu de sons propres à enchanter l'oreille du récepteur.

Le retour aux formes du lyrisme hellénique intéresse les poèmes de l'époque révolutionnaire (odes, hymnes, iambes) et soulève à leur propos des problèmes spécifiques d'ordre métrique et prosodique, voire syntaxique. Signe supplémentaire de la souplesse d'un génie réceptif aux modes sans pour autant devenir leur esclave. Homme de son temps, poète du souffle et du cœur, Chénier a pratiqué une écriture «sensorielle» dont le corollaire obligé est la lecture «auditive» (à haute voix) à laquelle est invité le lecteur mis en présence d'une écriture dans son éclosion primitive. S'il veut bien jouer le jeu, ce dernier pénètrera plus qu'auparavant dans l'atelier du fondeur, découvrira avec enchantement les secrets d'une alchimie, entendra en direct les inflexions, la respiration du poète. Faut-il, une fois de plus, déplorer la perte en 1870 d'une bonne part des papiers auxquels l'auteur tenait tant? Pour ces textes-là, faute de mieux, il est logique de retenir la version présumée la moins infidèle qui est en général celle de l'édition princeps. Malgré les retouches dont il a été accusé trop sévèrement, il se peut aussi que les leçons proposées par Latouche aient conservé quelques vestiges des particularités de l'original: il serait malvenu de les détruire.

Méconnaître toutes ces incidences au nom de principes que l'on croyait périmés, n'est-ce pas trahir l'ouvrage du poète et risquer de tromper l'amateur de poésie? C'est en tout cas la seule réserve sérieuse que l'on peut exprimer à l'encontre de l'édition des $O P 1$, dont la réalisation est si remarquable par ailleurs qu'elle a recueilli des éloges très mérités. À propos de la ponctuation qu'il a décidé de modifier, les arguments avancés par l'éditeur pour se justifier surprennent. Il rappelle «que Chénier n'a pratiquement pas publié de son vivant et que la plupart des manuscrits qui nous sont parvenus n'étaient pas destinés à être publiés en l'état» (OP1, p. 106). Que l'état des textes laissés par le poète à sa mort soit provisoire, personne n'en doute. Cela autorise-t-il pour autant des mains étrangères à «inventer» un état définitif forcément arbitraire et infidèle? Toute la question est là. Certains précédents en la matière devraient inciter à la prudence, car modifier revient toujours à falsifier. Les premiers éditeurs de La Jeune Tarentine ont imposé aux vers une toilette qui a fait écrire à Joubert dans son journal: «L'impression l'embellit» ${ }^{24}$. Nul n'en juge de même aujourd'hui. Sur le manuscrit de l'Hymne à la nuit le deuxième alexandrin est incomplet; une main étrangère a écrit les syllabes manquantes, ce qui donne le mélange suivant:

Salut, ô belle nuit, étincelante et sombre,

Consacrée au repos, ô silence de l'ombre $[. .$.

(D. II, p. 105)

La platitude de l'hémistiche le condamne d'emblée. Mais la tentation de jouer avec les textes taquinait les esprits à l'époque romantique. Le vers final manque à l'une des odes à Fanny aux allures d'odelette: elle est écrite en octosyllabes suivis et le vers absent rime avec le mot contour. Sainte-Beuve, Boissonade, Becq de Fouquières

(24) Voir sur la question G. BuIsson, Joseph Joubert et André Chénier, Actes du colloque Joubert de 1991 à Montignac, Villeneuve-sur-Yonne, 1995, pp. 31-45. 
entre autres se sont ingéniés à le restituer; le résultat n'est pas convaincant ${ }^{25}$. En 1864 Anatole France a fait paraître un prétendu inédit de Chénier qu'il s'était amusé à fabriquer: plusieurs lecteurs éminents s'y sont laissés prendre, à commencer par Becq de Fouquières qui l'a inséré dans sa réédition de 1872 (pp. 135-136).

Les retouches apportées à la ponctuation sont-elles de moindre conséquence que les attentats commis sur le texte même? Voire! Une virgule ajoutée, déplacée ou supprimée, un point-virgule substitué à un point (ou l'inverse) peuvent modifier notablement l'allure d'une phrase jusqu'à abolir le phrasé du poète comme nous le montrerons par des exemples dans un article à venir. Dans la seule Jeune Tarentine, où il a dénombré «vingt-sept fautes pour trente vers», Heredia dénonce avec beaucoup de pertinence «les plus fâcheux effets» de ce qu'il appelle «cette manie absurde» ${ }^{26}$. L'éditeur des OP1 dit encore avoir préféré

s'inspirer de la méthode de Paul Dimoff, de crainte qu'une ponctuation conforme en toute occasion aux données initiales dont on dispose ne nuise à la lisibilité et à la cohérence de l'ensemble. (p. 106)

Voilà qui s'appelle avancer à reculons. Étrange caution et crainte surprenante! Convient-il de verser du vin neuf dans des outres vieillies et usagées? La notion de cohérence du texte ressortit à une logique d'achèvement que les faits contredisent. Le travail inabouti du poète nous a transmis un ensemble inachevé, donc forcément disparate: devra-t-on lui imposer une cohérence forcée? Avec la méthode «dimovienne», qui relègue en bas de page et en caractères minuscules les indications à partir desquelles on peut rétablir le texte originel, l'utilisateur est condamné à une contorsion permanente et incommode s'il veut lire l'authentique production du poète... à moins de s'armer d'un effaceur et de supprimer tous les signes intrus (je connais des amateurs exigeants qui l'ont fait).

Sur la notion de lisibilité, il faudrait s'expliquer. L'éditeur invoque un «souci de clarté pour des lecteurs contemporains» (p. 106). Veut-on guider lesdits lecteurs pas à pas en respectant un mot à mot de nature assez primaire? Un tutorat de cette espèce n'a pas sa place dans une édition critique qui, par destination, ne s'adresse pas à un public de «mal lisants». Édition savante ou édition populaire, il faut choisir. Celle-ci, par son volume, par son prix, par la science éblouissante dont elle fait preuve à chaque page, par la richesse surabondante de ses commentaires, par sa rigueur, sa précision, son acribie, n'appartient évidemment pas à la seconde catégorie. En revanche, exclure le respect de la ponctuation de cette réalisation herculéenne - pour des motifs pour le moins flottants (voir OP1, p. 103) - relève d'un manque de cohérence peu compréhensible. C'est laisser croire à tort au lecteur que le poète plaçait la grammaire au-dessus des exigences rythmiques et prosodiques. C'est éliminer tout un arrière-fond historique. C'est ignorer les inflexions spécifiques d'un langage à nul autre semblable. C'est réduire à l'état de prose une poésie que caractérise avant toute chose sa musicalité.

Le paradoxe d'un tel parti pris déconcerte d'autant plus que l'attention portée à ce qui est du ressort de la bibliographie matérielle est poussée au maximum: sur l'épaisseur, l'origine, la couleur et la dimension des papiers, sur le nombre et l'écartement des vergeures et des pontuseaux, sur la nature des filigranes, sur les déchirures éventuelles du papier et sur la taille des trous, etc. aucune précision ne nous est épargnée. Le nombre des points de suspension utilisés par Chénier (il varie de deux à

(25) Voir Sainte-Beuve, éd. cit., p. 142 et l'édition publiée en 1872 par BECQ DE FOUQuiÈres, pp.
290 et 291.

(26) Les Bucoliques, éd. cit., p. XXIII. 
une dizaine) est chaque fois reproduit avec le plus grand soin. La moindre rature est interprétée. On ne peut que se réjouir d'une telle acribie qui profite à la datation et au classement du moindre «élément textuel» et déplorer la mise sur la touche des indices aussi substantiels que fournit le «système ponctuant» du poète. Le commentaire immédiat et les exégèses à venir ne pouvaient que se trouver enrichis de leur présence alors que leur évincement les appauvrit d'autant.

L'actualité nous fournit un ultime exemple de cette disposition d'esprit. Un événement rare dans la vie posthume du poète s'est produit récemment: un manuscrit d'André Chénier a été retrouvé par Catriona Seth dans les fonds de la Nationalbibliothek de Vienne. Il s'agit d'un petit morceau de vergé de 8,6 cm de long sur 3,8 de large contenant six alexandrins autographes qui offrent une variante inédite de vers connus. Pas moins de sept pages de commentaire lui sont consacrées, dues aux soins conjugués de C. Seth et de Georges Buisson ${ }^{27}$. Elles passionneront le petit nombre des spécialistes seuls capables d'en saisir la portée et l'intérêt. L'enquête sur les aspects historiques (circonstances de l'acheminement du fragment jusqu'à sa dernière destination) et critiques (comparaison minutieuse de la version retrouvée avec les versions déjà connues) est d'une exhaustivité qui force l'admiration; elle n'exclut pas des «calculs compliqués» (p. 155, note 10) à propos des mensurations au millimètre près du papier viennois confrontées aux données du manuscrit Brölemann. Dans ce luxe d'érudition plus que savante, propre à donner le vertige aux non-initiés, on cherche vainement la moindre trace de commentaire consacré à la ponctuation pourtant très «particulière», très instructive et tout à fait susceptible d'intéresser un large public d'amateurs éclairés, du fragment reproduit p. 152. Ainsi se confirme la méconnaissance systématique d'un des aspects d'une écriture (à tous les sens du mot) qui n'est pas, loin de là, le moins attrayant du point de vue philologique et littéraire. Ce rejet est-il justifié? Les analyses comparatives auxquelles nous procéderons dans une étude à venir permettront à chacun de juger sur pièces.

ÉDOUARD GUITTON

(27) C. SEтH, Un manuscrit d'André Chénier retrouvé, «Cahiers Roucher-André Chénier», n²7, 